\title{
AN EXTENSION OF \\ THE FUGLEDE COMMUTATIVITY THEOREM MODULO THE HILBERT-SCHMIDT CLASS TO OPERATORS OF THE FORM $\sum M_{n} X N_{n}$
}

BY

GARY WEISS

\begin{abstract}
We study the operators $\Delta(X)=\Sigma_{1}^{n} M_{n} X N_{n}$ and $\Delta^{*}(X)=\sum_{1}^{n} M_{n}^{*} X N_{n}^{*}$ which map the algebra of all bounded linear operators on a separable Hilbert space to itself, where $\left\langle M_{n}\right\rangle_{1}^{m}$ and $\left\langle N_{n}\right\rangle_{1}^{m}$ are separately commuting sequences of normal operators. We prove that (1) when $m \leqslant 2$, the Hilbert-Schmidt norms of $\Delta(X)$ and $\Delta^{*}(X)$ are equal (finite or infinite); (2) for $m \geqslant 3$, if $\Delta(X)$ and $\Delta^{*}(X)$ are Hilbert-Schmidt operators, then their Hilbert-Schmidt norms are equal; (3) if $\Delta, \Delta^{*}$ have the property that for each $X, \Delta(X)=0$ implies $\Delta^{*}(X)=0$, then for each $X$, if $\Delta(X)$ is a Hilbert-Schmidt operator then $\Delta^{* 2}(X)$ is also and the latter has the same Hilbert-Schmidt norm as $\Delta^{2}(X)$. Note that Fuglede's Theorem is immediate from (1) in the case $m=2, M_{1}=N_{2}$ and $N_{1}=I=-M_{2}$. The proofs employ the duality between the trace class and the class of all bounded linear operators and, unlike the early proofs of Fuglede's Theorem, they are free of complex function theory.
\end{abstract}

Let $H$ denote a separable, finite-dimensional, complex Hilbert space. Let $L(H) \supset$ $K(H) \supset C_{p} \supset F(H), C_{2}, C_{1},\|\cdot\|_{p},\|\cdot\|$ denote, respectively, the class of all bounded linear operators acting on $H$, the compact operators, the Schatten $p$-class, the finite rank operators, the Hilbert-Schmidt class, the trace class, the $C_{p}$-norm, and the operator norm.

Definition. Let $\left\langle M_{n}\right\rangle_{n=1}^{m}$ and $\left\langle N_{n}\right\rangle_{n=1}^{m}$ be two separately commuting sequences of normal operators. Define $\Delta, \Delta^{*}: L(H) \rightarrow L(H)$ by

$$
\Delta(X)=\sum_{n=1}^{m} M_{n} X N_{n} \text { and } \Delta^{*}(X)=\sum_{n=1}^{m} M_{n}^{*} X N_{n}^{*} .
$$

We shall study the algebra operator $\Delta: L(H) \rightarrow L(H)$ to determine conditions when $\Delta$ is invertible and when it is 1-1. Our Main Theorem generalizes [8, Theorem 4] to the operator $\Delta$.

MAIN Theorem 1. Let $\Delta, \Delta^{*}$ be as defined above. If $\Delta(X), \Delta^{*}(X) \in C_{2}$, then

$$
\|\Delta(X)\|_{2}=\left\|\Delta^{*}(X)\right\|_{2} \text {. }
$$

Received by the editors December 5, 1980.

1980 Mathematics Subject Classification. Primary 47A62, 47B10, 47B15; Secondary 47A65, 47B05, $47 \mathrm{~B} 47$.

Key words and phrases. $\Delta$-operator, Hilbert-Schmidt operator, trace class operator, commutator, duality, reproducing kernel function.

${ }^{1}$ This research was partially supported by a grant from N.S.F. 
Problem 1. (a) Does $\Delta(X) \in C_{2}$ imply $\Delta^{*}(X) \in C_{2}$ ?

(b) Does $\Delta(X)=0$ imply $\Delta^{*}(X)=0$ ?

1. Motivation. The Fuglede-Putnam Theorem states that if $N_{1}, N_{2}$ are normal operators and $X \in L(H)$, then $N_{1} X-X N_{2}=0$ implies $N_{1}^{*} X-X N_{2}^{*}=0$. The Main Theorem and Problem 1 show that Fuglede's Theorem is part of a more general phenomenon. Namely the more general expressions of $\sum M_{n} X N_{n}$ are involved together with their Hilbert-Schmidt norms.

In [9] we proved Problem 1 for the case $m=2$. This will serve as a first step in our induction proof for Theorem 1.

In [7] Voiculescu proved that if $S_{1}, \ldots, S_{k}$ are commuting selfadjoint operators, then there are diagonal operators $D_{1}, \ldots, D_{k}$ and a unitary operator $U$ such that for $n=1, \ldots, k, U S_{n} U^{-1}-D_{n} \in C_{k}$ with arbitrarily small $C_{k}$-norm. In addition, this result is sharp in that $C_{k}$ is the smallest $C_{p}$-class for which we can insure that all the $U S_{n} U^{-1}-D_{n} \in C_{p}$.

We pointed out in [9] that Voiculescu's result implies the results in [9]. However, even though his result is sharp, still if all the $M_{n}$ 's and $N_{n}$ 's are all diagonal (or arbitrarily small $C_{2}$ perturbations of simultaneously diagonalizable operators), then a straightforward computation shows that our Main Theorem holds true in this case where Voiculescu's result does not. It has been, and continues to be, of interest to ask what properties that would follow if every commuting family of selfadjoint operators were simultaneously Hilbert-Schmidt perturbations of diagonal operators are true anyway. Expressed more naively, in what ways are commuting normal operators like diagonal operators. The Main Theorem is one such way.

The first lemma uses Voiculescu's Theorem and provides us with some motivation. It also illustrates the limitations of his theorem in yielding a result like the Main Theorem or Problem 1.

LEMMA 1. If $\left\langle M_{n}\right\rangle_{1}^{m},\left\langle N_{n}\right\rangle_{1}^{m}$ are separately commuting sequences of normal operators and $X \in C_{p}$, where $1 / p+1 / 2 m=1 / 2$, then

$$
\left\|\sum_{1}^{m} M_{n} X N_{n}\right\|_{2}=\left\|\sum_{1}^{m} M_{n}^{*} X N_{n}^{*}\right\|^{2} .
$$

Proof. Let $M_{n}^{\prime}=M_{n} \oplus N_{n}$ and $Y=\left(\begin{array}{ll}0 & X \\ 0 & 0\end{array}\right)$ which act on the Hilbert space $H \oplus H$. Then a straightforward computation gives

$$
\left\|\sum_{1}^{m} M_{n} X N_{n}\right\|_{2}=\left\|\sum_{1}^{m} M_{n}^{\prime} Y M_{n}^{\prime}\right\|_{2}
$$

and

$$
\left\|\sum_{1}^{m} M_{n}^{*} X N_{n}^{*}\right\|_{2}=\left\|\sum_{1}^{m} M_{n}^{\prime *} Y M_{n}^{\prime *}\right\|_{2} .
$$

This proves that to prove Theorem 1 or Lemma 1 (or to settle Problem 1(a) or (b)), it suffices to prove the case $M_{n}=N_{n}$. 
Applying Voiculescu's Theorem to the real and imaginary parts of $M_{n}=N_{n}$, which form a family of $2 m$ commuting selfadjoint operators, we can obtain a unitary operator $U$, diagonal operators $D_{1}, \ldots, D_{m}$ with $\left\|D_{n}\right\| \leqslant 2\left\|M_{n}\right\|$ for each $n$, and compact operators $K_{1}, \ldots, K_{m} \in C_{2 m}$ with $\left\|K_{n}\right\|_{2 m}<\varepsilon /\left(6 m \max \left\|M_{n}\right\|^{2}\right)$. Clearly if we let $Y=U X U^{*}$ then $\|Y\|_{p}=\|X\|_{p}$ and

$$
\left\|\sum_{1}^{m} M_{n} X N_{n}\right\|_{2}=\left\|\sum_{1}^{m} D_{n} Y D_{n}+\sum_{1}^{m} K_{n} Y K_{n}+\sum_{1}^{m} K_{n} Y D_{n}+\sum_{1}^{m} D_{n} Y K_{n}\right\|_{2}
$$

and

$$
\left\|\sum_{1}^{m} M_{n}^{*} X M_{n}^{*}\right\|_{2}=\left\|\sum_{1}^{m} D_{n}^{*} Y D_{n}^{*}+\sum_{1}^{m} K_{n}^{*} Y K_{n}^{*}+\sum_{1}^{m} K_{n}^{*} Y D_{n}^{*}+\sum_{1}^{m} D_{n}^{*} Y K_{n}^{*}\right\|_{2} .
$$

But

$$
\left\|\sum_{1}^{m} K_{n} Y K_{n}+\sum_{1}^{m} K_{n} Y D_{n}+\sum_{1}^{m} D_{n} Y K_{n}\right\|_{2} \leqslant \varepsilon
$$

by Hölder's inequality in the Schatten classes where $1 / p+1 / 2 m=1 / 2$ [2]. Similarly for the * counterpart. Since $\varepsilon$ is arbitrary, it clearly suffices to prove

$$
\left\|\sum_{1}^{m} D_{n} Y D_{n}\right\|_{2}=\left\|\sum_{1}^{m} D_{n}^{*} Y D_{n}^{*}\right\|_{2} \text {. }
$$

Now let $\left\langle\lambda_{n}(k)\right\rangle_{k=1}^{\infty}$ denote the diagonal entries of the diagonal matrix $D_{n}$ and let $y_{i j}$ denote the matrix entries of $Y$. Then a straightforward computation shows that the $(i, j)$ th entry of $\sum_{1}^{m} D_{n} Y D_{n}$ is $\left(\sum_{n=1}^{m} \lambda_{n}(i) \lambda_{n}(j)\right) y_{i j}$ and that of $\sum_{1}^{m} D_{n}^{*} Y D_{n}^{*}$ is $\left(\sum_{n=1}^{m} \overline{\lambda_{n}(i) \lambda_{n}(j)}\right) y_{i j}$. Since both these entries have equal absolute values and the Hilbert-Schmidt norms of these operators can be expressed as the sum over $i, j$ of the squares of the absolute values of these numbers, the above equality holds. Q.E.D.

Lemma 1 provides us with two corollaries we shall need later.

Corollary 2. The Main Theorem holds true when $X \in C_{2}$.

Proof. Apply Lemma 1. Note that if $1 / p+1 / 2 m=1 / 2$ then $p>2$, hence $C_{2} \subset C_{p}$.

Corollary 3. The Main Theorem is equivalent to the case where $M_{n}=N_{n}$.

Proof. See the proof of Lemma 1 (first paragraph).

REMARK. Lemma 1 can be strengthened to hold for $C_{p}$ where $1 / p+1 / 2(m-1)$ $=1 / 2$. The argument is similar to the proof of Lemma 4 , reducing to the case where $M_{1}$ is invertible, then eliminating it to where the number of normals is reduced by one.

2. A distribution theory for operators. This section provides us with a technical tool.

In [8] the author introduced generating functions and test functions in connection with operators. This treatise avoids direct use of generating functions, replacing 
them with their operator theoretic counterparts. However, we could not avoid the distribution structure altogether.

It is difficult to motivate this section before seeing the need for it arise in a proof. In any case we attempt a partial motivation.

Later we shall encounter an operator $T$ for which $(T f, g)=0$ for certain $f, g \in$ $L^{2}[0,1]$. We will want to show $T=0$. It is well known and elementary to verify that $(T f, g)=\operatorname{trace} T(f \otimes g)$ where $f \otimes g$ is the rank one operator $h \rightarrow(, g) f$. This phenomenon is related to the fact that the dual of $C_{1}$ is $L(H)$. It is this point of view that has led the author to many of the results in this area.

Therefore one might wish to decide if all the $f, g$ for which $(T f, g)=0$ when used to form $f \otimes g \operatorname{span} C_{1}$. This would give trace $T K=0$ for all $K \in C_{1}$. Hence $(T f, g)=\operatorname{trace} T(f \otimes g)=0$ for all $f, g \in L^{2}[0,1]$, so $T$ would equal 0 .

We need this sort of consideration regarding $\Delta$ and $\Delta^{*}$.

Lemma 4 (The Key Lemma). Suppose $m=2$ with respect to $\Delta$. Fix $K \in C_{1}$ and $\varepsilon>0$.

(a) If $M_{2}$ and $N_{1}$ are invertible, then there exists $\tilde{K} \in C_{1}$ such that

$$
\left\|\Delta(K)-\Delta^{*}(\tilde{K})\right\|_{1}<\varepsilon .
$$

(b) There exists $\tilde{K} \in C_{1}$ such that $\left\|\Delta(K)-\Delta^{*}(\tilde{K})\right\|_{1}<\varepsilon$.

(c) $C_{1}$-closure of $\Delta\left(C_{1}\right)=C_{1}$-closure of $\Delta^{*}\left(C_{1}\right)$. Equivalently, the $C_{1}$-closure of $\Delta\left(C_{1}\right)$ is selfadjoint.

Proof. Clearly (b) implies (c) since $\Delta, K$ and $\varepsilon$ are arbitrary. We first show that (a) implies (b). Then we show that (a) is true.

Consider a spectral representation for the commuting normal operators $M_{1} \cong M_{\phi}$ and $M_{2} \cong M_{\psi}$ taking place on a finite regular borel measure space. Let $\chi_{\varepsilon}=$ $\chi_{\{x:|\psi(x)|>\varepsilon\}}$ and let $P_{\varepsilon}$ denote the projections corresponding to $M_{x_{\varepsilon}}$. Then $P_{\varepsilon} \uparrow P_{0}$ in the strong operator topology as $\varepsilon \rightarrow 0$. Set

$$
\alpha(x)= \begin{cases}\frac{\overline{\phi(x)}}{\phi(x)} & \text { if } \phi(x) \neq 0, \\ 0 & \text { if } \phi(x)=0,\end{cases}
$$

and

$$
\beta(x)= \begin{cases}\overline{\psi(x)} & \text { if } \psi(x) \neq 0, \\ 0 & \text { if } \psi(x)=0 .\end{cases}
$$

Let $A_{1}$ denote the partial isometry corresponding to $M_{\alpha}$ and $A_{2}$ the partial isometry corresponding to $M_{\beta}$. Hence $M_{1}, M_{2}, P_{\varepsilon}, P_{0}, A_{1}, A_{2}$ all commute and, in addition, $A_{1} M_{1}=M_{1}^{*}, A_{2} M_{2}=M_{2}^{*},\left(1-P_{0}\right) A_{1}=0$, and $M_{2}\left(1-P_{0}\right)=0$. Similarly for $N_{1}$, $N_{2}$ there exist $B_{1}, B_{2}$ and projections $Q_{\varepsilon} \uparrow Q_{0}$ in the strong operator topology such that $N_{1}, N_{2}, Q_{\varepsilon}, Q_{0}, B_{1}, B_{2}$ all commute, and, in addition,

$$
B_{1} N_{1}=N_{1}^{*}, \quad B_{2} N_{2}=N_{2}^{*}, \quad B_{1}\left(1-Q_{0}\right)=0, \quad \text { and } \quad\left(1-Q_{0}\right) N_{1}=0 \text {. }
$$


Now fix $K \in C_{1}$. Then

$$
\begin{aligned}
K= & \left(1-P_{0}\right) K+\left(P_{0}-P_{\varepsilon}\right) K+P_{\varepsilon} K \\
= & \left(1-P_{0}\right)\left[K\left(1-Q_{0}\right)+K\left(Q_{0}-Q_{\varepsilon}\right)+K Q_{\varepsilon}\right] \\
& +\left(P_{0}-P_{\varepsilon}\right)\left[K\left(1-Q_{0}\right)+K\left(Q_{0}-Q_{\varepsilon}\right)+K Q_{\varepsilon}\right] \\
& +P_{\varepsilon}\left[K\left(1-Q_{0}\right)+K\left(Q_{0}-Q_{\varepsilon}\right)+K Q_{\varepsilon}\right] \\
= & \left(1-P_{0}\right) K\left(1-Q_{0}\right)+\left(1-P_{0}\right) K\left(Q_{0}-Q_{\varepsilon}\right)+\left(1-P_{0}\right) K Q_{\varepsilon} \\
& +\left(P_{0}-P_{\varepsilon}\right) K\left(1-Q_{0}\right)+\left(P_{0}-P_{\varepsilon}\right) K\left(Q_{0}-Q_{\varepsilon}\right)+\left(P_{0}-P_{\varepsilon}\right) K Q_{\varepsilon} \\
& +P_{\varepsilon} K\left(1-Q_{0}\right)+P_{\varepsilon} K\left(Q_{0}-Q_{\varepsilon}\right)+P_{\varepsilon} K Q_{\varepsilon} .
\end{aligned}
$$

Since $P_{\varepsilon} \rightarrow P_{0}$ and $Q_{\varepsilon} \rightarrow Q_{0}$ in the strong operator topology as $\varepsilon \rightarrow 0$, as is well known, for every $T \in C_{1}$ we have $\left(P_{\varepsilon}-P_{0}\right) T \rightarrow 0$ and $T\left(Q_{\varepsilon}-Q_{0}\right) \rightarrow 0$ in the trace norm. Therefore in the last equality, each term with $P_{0}-P_{\varepsilon}$ or $Q_{0}-Q_{\varepsilon}$ as a factor tends to 0 in the trace norm.

Since $\left(1-P_{0}\right) M_{2}=0=N_{1}\left(1-Q_{0}\right)$, if we apply $\Delta$ to the terms in the last equality with $1-P_{0}$ or $1-Q_{0}$ as a factor, we obtain a 1-sum rather than the usual 2-sum. For example,

$$
\Delta\left(\left(1-P_{0}\right) K\left(Q_{0}-Q_{\varepsilon}\right)\right)=M_{1}\left(1-P_{0}\right) K\left(Q_{0}-Q_{\varepsilon}\right) N_{1} .
$$

Hence

$$
\Delta(K)=M_{1}\left(1-P_{0}\right) K Q_{\varepsilon} N_{1}+M_{2} P_{\varepsilon} K\left(1-Q_{0}\right) N_{2}+\Delta\left(P_{\varepsilon} K Q_{\varepsilon}\right)+T_{\varepsilon}
$$

where $\Delta\left(\left(1-P_{0}\right) K\left(1-Q_{0}\right)\right)=0$ and $T_{\varepsilon}$ is the sum of all the terms with $P_{0}-P_{\varepsilon}$ or $Q_{0}-Q_{\varepsilon}$ as a factor. Hence $T_{\varepsilon} \rightarrow 0$ in the trace norm as $\varepsilon \rightarrow 0$.

Set $\tilde{K}_{1}=A_{1}^{*}\left(1-P_{0}\right) K Q_{\varepsilon} B_{1}^{*}$ and $\tilde{K}_{2}=A_{2}^{*} P_{\varepsilon} K\left(1-Q_{0}\right) B_{2}^{*}$. Note that $M_{1}^{*} A_{1}^{*}=M_{1}$ and $B_{1}^{*} N_{1}^{*}=N_{1}$ (since $M_{1} A_{1}=M_{1}^{*}$ and $B_{1} N_{1}=N_{1}^{*}$ ). Therefore

$$
\Delta^{*}\left(\tilde{K}_{1}\right)=M_{1}^{*} A_{1}^{*}\left(1-P_{0}\right) K Q_{\varepsilon} B_{1}^{*} N_{1}^{*}+0=M_{1}\left(1-P_{0}\right) K Q_{\varepsilon} N_{1} .
$$

Similarly $\Delta^{*}\left(\tilde{K}_{2}\right)=M_{2} P_{\varepsilon} K\left(1-Q_{0}\right) N_{2}^{*}$. Clearly we also have $\tilde{K}_{1}, \tilde{K}_{2} \in C_{1}$.

To deal with $\Delta\left(P_{\varepsilon} K Q_{\varepsilon}\right)$, define $M_{2}^{\prime}=M_{2} P_{\varepsilon}+\left(1-P_{\varepsilon}\right) \cong M_{\psi x_{\varepsilon}+\left(1-x_{\varepsilon}\right.}$. Since $\psi x_{\varepsilon}$ $+\left(1-x_{\varepsilon}\right)$ is bounded below, $M_{2}^{\prime}$ is normal and invertible. Similarly defining $N_{1}^{\prime}=Q_{\varepsilon} N_{1}+\left(1-Q_{\varepsilon}\right)$, we have $N_{1}^{\prime}$ is normal and invertible. Because $P_{\varepsilon}\left(1-P_{\varepsilon}\right)=0$ $=Q_{\varepsilon}\left(1-Q_{\varepsilon}\right)$ we have $\Delta\left(P_{\varepsilon} K Q_{\varepsilon}\right)=M_{1} P_{\varepsilon} K Q_{\varepsilon} N_{1}^{\prime}+M_{2}^{\prime} P_{\varepsilon} K Q_{\varepsilon} N_{2}$, but the right-hand side of this expression satisfies the hypotheses of part (a). Fixing $\varepsilon_{0}>0$ and assuming (a) for the moment, we obtain $\tilde{K}_{3}^{\prime} \in C_{1}$ such that if we denote $\Delta^{\prime}(X)=$ $M_{1} X N_{1}^{\prime}+M_{2}^{\prime} X N_{2}$ and similarly for $\Delta^{\prime *}(X)$, we have $\left\|\Delta^{\prime}\left(P_{\varepsilon} K Q_{\varepsilon}\right)-\Delta^{\prime *}\left(\tilde{K}_{3}^{\prime}\right)\right\|_{1}<$ $\varepsilon_{0} / 2$ with $\Delta\left(P_{\varepsilon} K Q_{\varepsilon}\right)=\Delta^{\prime}\left(P_{\varepsilon} K Q_{\varepsilon}\right)$. Set $\tilde{K}_{3}=P_{\varepsilon} \tilde{K}_{3}^{\prime} Q_{\varepsilon}$. Then $\Delta^{*}\left(\tilde{K}_{3}\right)=\Delta^{\prime *}\left(\tilde{K}_{3}^{\prime}\right)$ and $\tilde{K}_{3} \in C_{1}$. So $\left\|\Delta\left(P_{\varepsilon} K Q_{\varepsilon}\right)-\Delta^{*}\left(\tilde{K}_{3}\right)\right\|_{1}<\varepsilon_{0} / 2$.

Finally set $\tilde{K}=\tilde{K}_{1}+\tilde{K}_{2}+\tilde{K}_{3}$, so $\tilde{K} \in C_{1}$ and $\|\Delta(K)-\Delta(\tilde{K})\|_{1}<\varepsilon_{0} / 2+\left\|T_{\varepsilon}\right\|_{1}$ (see $(*))$. But $\varepsilon$ is arbitrary and hence we can choose it close enough to 0 such that $\left\|T_{\varepsilon}\right\|_{1}<\varepsilon_{0} / 2$. That is, $\left\|\Delta(K)-\Delta^{*}(\tilde{K})\right\|_{1}<\varepsilon_{0}$. This completes the proof that (a) implies (b).

To prove (a) we first reduce to the case that $\Delta(X)=M X-X N$ with $M, N$ normal (not necessarily commuting). To see this, first observe that there is clearly no loss in 
generality if we replace + in $\Delta$ and $\Delta^{*}$ by - . Now $M_{2}, N_{1}$ invertible imply easily that $M_{2}^{-1} M_{2}^{*}$ and $N_{1}^{-1} N_{1}^{*}$ are unitary operators and so, as is well known, they preserve the trace norm (that is, if $H$ is a unitary operator then $\|U T\|_{1}=\|T\|_{1}$ for every $T \in C_{1}$ ).

Now,

$$
\Delta(K)=M_{2}\left(M_{2}^{-1} M_{1} K-K N_{2} N_{1}^{-1}\right) N_{1}
$$

and $M_{2}^{-1} M_{1} K-K N_{2} M^{-1}$ is of the form $M X-X N$ with $M, N$ normal. If we assume that (a) holds for these forms, then there exists $\tilde{K}_{1} \in C_{1}$ such that

$$
\left\|M_{2}\right\|\left\|\left(M_{2}^{-1} M_{1} K-K N_{2} N_{1}^{-1}\right)-\left(M_{2}^{-1^{*}} M_{1}^{*} \tilde{K}_{1}-\tilde{K}_{1} N_{2}^{*} N_{1}^{-1^{*}}\right)\right\|_{1}\left\|N_{1}\right\|<\varepsilon .
$$

But

$$
\begin{gathered}
M_{2}\left(M_{2}^{-1^{*}} M_{1}^{*} \tilde{K}_{1}-\tilde{K}_{1} N_{2}^{*} N_{1}^{-1^{*}}\right) N_{1}=M_{2}^{*} M_{2}^{-1^{*}} M_{2}\left(M_{2}^{-1^{*}} M_{1}^{*} \tilde{K}_{1}-\tilde{K}_{1} N_{2}^{*} N_{1}^{-1^{*}}\right) N N_{1}^{-1^{*}} N_{1}^{*} \\
=M_{1}^{*}\left(M_{2}^{-1^{*}} M_{2} \tilde{K}_{1} N_{1} N_{1}^{-1^{*}}\right) N_{1}^{*}-M_{2}^{*}\left(M_{2}^{-1^{*}} M_{2} \tilde{K}_{1} N_{1} N_{1}^{-1^{*}}\right) N_{2}^{*} .
\end{gathered}
$$

Set $\tilde{K}=M_{2}^{-1^{*}} M_{2} \tilde{K}_{1} N_{1} N_{1}^{-1^{*}}$. Then $\tilde{K} \in C_{1}$. Then putting this all together we have $\left\|\Delta(K)-\Delta^{*}(\tilde{K})\right\|_{1}<\varepsilon$. Hence we may assume $\Delta(X)=M X-X N$.

Next we reduce to the case $\Delta(X)=N X-X N$ where $N$ is normal. Set $H^{\prime}=H \oplus H$, $N^{\prime}=M \oplus N$ and $\Delta^{\prime}(X)=N^{\prime} X-X N^{\prime}$ for $X \in H^{\prime}$. Set $K^{\prime}=\left(\begin{array}{ll}0 & K \\ 0 & 0\end{array}\right), K \in C_{1}$. Then

$$
\Delta^{\prime}\left(K^{\prime}\right)=\left(\begin{array}{cc}
0 & \Delta(K) \\
0 & 0
\end{array}\right)
$$

and similarly for $\Delta^{\prime *}\left(K^{\prime}\right)$. If we assume (a) when $M=N$, that is, for $\Delta^{\prime}$, then there exists $\tilde{K}^{\prime} \in C_{1}$ (relative to $\left.H \oplus H\right)$ such that $\left\|\Delta^{\prime}\left(K^{\prime}\right)-\Delta^{\prime *}\left(K^{\prime}\right)\right\|_{1}<\varepsilon$. But

$$
\tilde{K}^{\prime}=\left(\begin{array}{cc}
* & \tilde{K} \\
* & *
\end{array}\right)
$$

relative to $H \oplus H$, and so $\tilde{K} \in C_{1}$. In addition,

$$
\left\|\Delta(K)-\Delta^{*}(\tilde{K})\right\|_{1}=\left\|\left(\begin{array}{cc}
0 & \Delta(K) \\
0 & 0
\end{array}\right)-\left(\begin{array}{cc}
0 & \Delta^{*}(\tilde{K}) \\
0 & 0
\end{array}\right)\right\|_{1} \leqslant\left\|\Delta^{\prime}\left(K^{\prime}\right)-\Delta^{\prime *}\left(\tilde{K}^{\prime}\right)\right\|_{1}<\varepsilon .
$$

(Compute $\Delta^{\prime *}\left(\tilde{K}^{\prime}\right)$ in terms of $\tilde{K}$.) Hence we may assume $M=N$.

To prove (a) when $\Delta(X)=N X-X N$, apply Voiculescu's Theorem to obtain a basis $\left\langle e_{n}\right\rangle_{1}^{\infty}$ in which $N=D+C$ where $D$ is diagonal and $C \in C_{2}$ with $\|C\|_{2}<\varepsilon$. Fixing $K \in C_{1}$, let $K(i, j)$ denote the matrix entries for $K$ with respect to this basis. Let $P_{n}$ denote the finite projection onto $\operatorname{span}\left\langle e_{1}, \ldots, e_{n}\right\rangle$ and set $K_{n}=P_{n} K P_{n}$. Hence

$$
K_{n}(i, j)= \begin{cases}K(i, j), & 1 \leqslant i, j \leqslant n, \\ 0 & \text { otherwise }\end{cases}
$$

and, as is well known, $K_{n} \in C_{1}$ and $K_{n} \rightarrow K$ in the trace norm. Therefore it suffices to prove (a) for $K_{n}$, since $\left\|\Delta(K)-\Delta\left(K_{n}\right)\right\|_{1} \rightarrow 0$. Let $\left\langle d_{n}\right\rangle_{1}^{\infty}$ denote the diagonal entries of $D$. Define

$$
\tilde{K}_{n}(i, j)= \begin{cases}\frac{d_{i}-d_{j}}{\bar{d}_{i}-\bar{d}_{j}} K_{n}(i, j) & \text { if } d_{i} \neq d_{j} \\ 0 & \text { otherwise }\end{cases}
$$


Then $\tilde{K}_{n}$ is a matrix whose entries are finitely nonzero and, hence, $\tilde{K}_{n} \in C_{1}$ with $\left\|\tilde{K}_{n}\right\|_{2}=\left\|K_{n}\right\|_{2} \leqslant\|K\|_{2} \leqslant\|K\|_{1}$. By computation $D K_{n}-K_{n} D=D^{*} \tilde{K}_{n}-\tilde{K}_{n} D^{*}$. Therefore

$$
\begin{aligned}
\left\|\Delta\left(K_{n}\right)-\Delta^{*}\left(\tilde{K}_{n}\right)\right\|_{1} & =\left\|\left(C K_{n}-K_{n} C\right)+\left(C^{*} \tilde{K}_{n}-\tilde{K}_{n} C\right)\right\| \\
& \leqslant 4\|C\|_{2}\|K\|_{2} \leqslant 4\|C\|_{2}\|K\|_{1}<4 \varepsilon\|K\|_{1} \text {. Q.E.D. }
\end{aligned}
$$

REMARK. In case this unified approach to these various Fuglede type theorems seems a bit circular, since the proof of the key lemma uses Voiculescu's new result, we wish to point out that this last reduction can be proved without Voiculescu's theorem using the techniques in [9] or Lemma 14 given later. However the proof is longer and more technical. For this reason we omit it.

Lemma 4 provides a new approach and a very short proof of the Fuglede-Putnam Theorem and, with no extra work, the extension to the $\Delta$-operator for $m=2$.

Theorem 5 (The Fuglede Theorem for the $\Delta$-operator with $m=2$ ). If $\Delta(X)=0$ then $\Delta^{*}(X)=0$.

Proof. Note that if $K$ is any trace class operator and $A, X, B \in L(H)$ then trace $A X B K=$ trace $X B K A$. This follows since if $T=X B K, T$ is a trace class operator and trace $A T=$ Trace $T A$, as is well known.

Let $\Delta_{1}(X)=\sum_{n=1}^{m} N_{n} X M_{n}$. Then by the above remarks, for every $K \in C_{1}$,

$$
\text { Trace } \Delta(X) K=\text { trace } X\left(\Delta_{1}(K)\right)
$$

and the same for $\Delta^{*}(X) K$.

Recall that for all $T \in L(H), f, g \in H$ we have $(T f, g)=\operatorname{trace} T(f \otimes g)$.

Now to show $\Delta^{*}(X)=0$, it suffices to show $\left(\Delta^{*}(X) f, g\right)=0$ for all $f, g \in H$. But

$$
\left(\Delta^{*}(X) f, g\right)=\operatorname{trace} \Delta^{*}(X)(f \otimes g)=\operatorname{trace} X\left[\Delta_{1}^{*}(f \otimes g)\right] .
$$

By Lemma 4 , for every $\varepsilon>0$ there exists $\tilde{K}$ such that $\left\|\Delta_{1}(\tilde{K})-\Delta_{1}^{*}(f \otimes g)\right\|_{1}<$ $\varepsilon /\|X\|$. Then

$$
\left|\operatorname{trace} X\left[\Delta_{1}^{*}(f \otimes g)\right]-\operatorname{trace} X\left[\Delta_{1}(\tilde{K})\right]\right| \leqslant\|X\|\left\|\Delta_{1}^{*}(f \otimes g)-\Delta_{1}(\tilde{K})\right\|_{1}<\varepsilon .
$$

But $\Delta X=0$ implies trace $X\left[\Delta_{1}(\tilde{K})\right]=\operatorname{trace}[\Delta(X)] \tilde{K}=0$. Therefore $\left|\left(\Delta^{*}(X) f, g\right)\right|$ $<\varepsilon$ for all $\varepsilon>0$. Since $\varepsilon$ was arbitrary, $\left(\Delta^{*}(X) f, g\right)=0$. Q.E.D.

REMARK. Here is the first use of the 'distribution theory for operators'. Using the operators $\Delta_{l}(K)$ to approximate $\Delta_{l}^{*}(f \otimes g)$ corresponds somewhat to the notion of test functions in the classical distribution theory.

3. Asymptotic Fuglede theorems. There has been a plethora of papers on asymptotic Fuglede theorems. The first, and perhaps most notable, is that of R. Moore [3] which states that if $N$ is normal and $\|X\| \leqslant M$, then for every $\varepsilon>0$ there exists $\delta>0$ such that $\|N X-X N\|<\delta$ implies $\left\|N^{*} X-X N^{*}\right\|<\varepsilon$.

D. Rogers [5] later proved similar asymptotic Fuglede theorems for the strong and weak operator topologies. He proved that if $\left\{X_{n}\right\} \subset L(H)$ are uniformly bounded then $N X_{n}-X_{n} N \rightarrow 0$ SOT (WOT) then $N^{*} X_{n}-X_{n} N^{*} \rightarrow 0$ SOT (WOT). 
We can give a proof for Rogers' result in the WOT using our approach of Lemma 4 and an alteration of the proof of Theorem 5.

THEOREM 6. Let $m=2$ for $\Delta$. If $\left\{X_{n}\right\}$ is uniformly bounded in $L(H)$, then $\Delta\left(X_{n}\right) \rightarrow 0(W O T)$ implies $\Delta^{*}\left(X_{n}\right) \rightarrow 0(W O T)$.

Proof. Note first that by the usual $2 \times 2$ matrix trick, we may assume that without loss of generality, $M_{n}=N_{n}$.

The proof of the theorem depends on the inequality from the proof of Theorem 5 . (Note: $\Delta_{1}=\Delta$, if $M_{n}=N_{n}$.)

$$
\begin{aligned}
\left|\left(\Delta^{*}(X) f, g\right)\right| & =\left|\left(\operatorname{trace} X\left[\Delta^{*}(f \otimes g)\right]-\operatorname{trace}[X \Delta(K)]\right)+\operatorname{trace}[X \Delta(K)]\right| \\
& \leqslant\|X\|\left\|\Delta^{*}(f \otimes g)-\Delta(K)\right\|_{1}+|\operatorname{trace}[\Delta(X) K]| .
\end{aligned}
$$

By hypothesis $\left\|X_{n}\right\| \leqslant M$ for some $M$ and all $n$.

A closer examination of our proof of Lemma 4 tells us that $\tilde{K}$ was actually chosen to be a finite rank operator. Hence we may assume here that $K=\Sigma_{1}^{N} f_{k} \otimes g_{k}$ and $\left\|\Delta^{*}(f \otimes g)-\Delta(K)\right\|_{1}<\varepsilon / 2 M$. Therefore by the above inequality

$$
\left|\left(\Delta^{*}\left(X_{n}\right) f, g\right)\right| \leqslant \frac{\varepsilon}{2}+\operatorname{tr} \Delta\left(X_{n}\right) K .
$$

But $\operatorname{tr} \Delta\left(X_{n}\right) K=\Sigma_{1}^{N}\left(\Delta\left(X_{n}\right) f_{k}, g_{k}\right) \rightarrow 0$ as $n \rightarrow \infty$, since $\left(\Delta\left(X_{n}\right) f_{k}, g_{k}\right) \rightarrow 0$ for each $k$. Therefore for $n$ sufficiently large, $\left|\left(\Delta^{*}\left(X_{n}\right) f, g\right)\right|<\varepsilon$. Q.E.D.

4. Reductions of the Main Theorem. In this section we prove several lemmas and theorems which reduce Problem 1 and our Main Theorem 1 to the case where $M_{n}=N_{n}=M_{\phi_{n}}$ (by Corollary 3), where in addition the $\Phi_{n}$ 's are continuous functions on $[0,1], H=L^{2}[0,1]$, and $\left\langle\phi_{n}\right\rangle$ are linearly independent on all measurable sets $E \subset[0,1]$ of positive measure. Then we develop the theory from this case.

The reader should keep in mind that there is an underlying induction hypothesis on $m$ throughout this section.

Lemma 7. Theorem 1 and Problem 1 are equivalent to the case $H=L^{2}(X, \mu)$ where $(X, \mu)$ is a regular borel probability measure on a compact Hausdorff space and for each $n, N_{n}=M_{n}=M_{\phi_{n}}$ where $\phi_{n} \in L^{\infty}(\mu)$.

Proof. Simply use a standard form of the spectral theorem applied to the commuting family of normal operators $\left\langle M_{n}\right\rangle_{1}^{m}$. That we can assume $M_{n}=N_{n}$ follows by Corollary 3 .

Crucial to the proof of Theorem 1 and a better understanding of Problem 1 is that $\left\langle M_{\phi_{n}}\right\rangle$ be linearly independent on all subspaces $L^{2}(E)$ where $E$ is measurable and $m E>0$. However this is not necessarily the case. We must first factor out the subspace of $L^{2}(E)$ where $M_{\phi_{n}}$ are linearly dependent and treat them separately.

Lemma 8. Theorem 1 (Problem 1) is equivalent to the case of Theorem 1 (Problem 1) where we assume in addition that for every measurable set $E$ with $m E>0$, the set $\left\langle M_{\phi_{n}}\right\rangle_{1}^{m}$ (each of which is reduced by $L^{2}(E)$ ) is linearly independent on $L^{2}(E)$.

Proof. For each nonzero $n$-tuple of complex numbers $\left(c_{1}, \ldots, c_{m}\right)$, let $E\left(c_{1}, \ldots, c_{m}\right)=\left\{x \in X: \sum_{n=1}^{m} c_{n} \phi_{n}(x)=0\right\}$. Clearly $E\left(c_{1}, \ldots, c_{m}\right)$ is measurable. 
Define $\alpha_{0}=\sup \left\{\mu\left[E\left(c_{1}, \ldots, c_{m}\right)\right]:\left(c_{1}, \ldots, c_{m}\right) \not 0\right\}$. Clearly $0 \leqslant \alpha_{0} \leqslant \mu X<\infty$. Now choose $E_{0}=E\left(c_{1}, \ldots, c_{m}\right)$ such that $\mu E_{0} \geqslant \alpha_{0} / 2$. Now inductively define $E_{n}$, $\alpha_{n}$ as follows. Let

$$
\alpha_{n}=\sup \left\{\mu\left[E\left(c_{1}, \ldots, c_{m}\right) \cap\left(X \backslash \bigcup_{0}^{n-1} E_{k}\right)\right]:\left(c_{1}, \ldots, c_{m}\right) \neq 0\right\} .
$$

Choose $E_{n}=\left[E\left(c_{1}, \ldots, c_{m}\right) \cap\left(X \backslash \cup_{0}^{n-1} E_{k}\right)\right]$ for some $\left(c_{1}, \ldots, c_{m}\right) \neq 0$ such that $\mu E_{n} \geqslant \alpha_{n} / 2$. The two pertinent facts here are firstly that the $E_{n}$ 's are disjoint and secondly that $\sum_{0}^{\infty} \alpha_{n} \leqslant 2 \sum \mu E_{n} \leqslant 2 \mu X<\infty$. Therefore $\alpha_{n} \rightarrow 0$ as $n \rightarrow \infty$.

Hence we have $X=E_{0} \bullet E_{1} \bullet \cdots \cup\left(X \backslash \cup_{0}^{\infty} E_{k}\right)$ and $\left\langle\phi_{n}\right\rangle_{1}^{m}$ are linearly dependent on each $E_{k}$ (hence $\sum_{1}^{m} c_{n} M_{\phi_{n}}=0$ on $L^{2}\left(E_{n}\right)$ for some fixed $\left.\left(c_{1}, \ldots, c_{m}\right) Z 0\right)$.

The essential claim here is that $X \backslash \cup_{0}^{\infty} E_{k}$ has the property that if $E$ is measurable, $E \subset X \backslash \cup_{0}^{\infty} E_{k}$ such that for some fixed $\left(c_{1}, \ldots, c_{m}\right) \not 0, \Sigma_{1}^{m} c_{n} \phi_{n}(x)=0$ on $E$, then $\mu E=0$. To see this, suppose to the contrary there is such a set $E$ with $\mu E>0$. But $E \subset X \backslash \cup_{0}^{\infty} E_{k} \subset X \backslash \cup_{0}^{n} E_{k}$. Hence by the definition of $\alpha_{n}, \alpha_{n} \geqslant \mu E$. This is a contradiction since $\alpha_{n} \rightarrow 0$.

Now set $E_{\infty}=X \backslash \cup_{0}^{\infty} E_{k}$. Then $H=\Sigma_{0}^{\infty} \oplus L^{2}\left(E_{k}\right) \oplus L^{2}\left(E_{\infty}\right)$ with each $L^{2}\left(E_{k}\right)$ $(0 \leqslant k \leqslant \infty)$ reducing all the $M_{n}$ 's. Let $P_{k}$ denote the orthogonal projection whose range is $L^{2}\left(E_{k}\right)$. It is easy to see that for every $Y \in L(H)$,

$$
\|Y\|_{2}^{2}=\sum_{i, j=0}^{\infty}\left\|P_{i} Y P_{j}\right\|_{2}^{2}
$$

where $i, j$ attain $\infty$ in this sum. Therefore to prove Theorem 1 it suffices to prove that for each $1 \leqslant i, j \leqslant \infty$,

$$
\left\|P_{i}\left[\sum_{1}^{m} M_{n} X M_{n}\right] P_{j}\right\|_{2}=\left\|P_{i}\left[\sum_{1}^{m} M_{n}^{*} X M_{n}^{*}\right] P_{j}\right\|_{2} .
$$

Note that if $i$ or $j<\infty$, say $i<\infty$, then $L^{2}\left(E_{i}\right)$ reduces the $M_{n}$ 's, and in fact there exists $\left(c_{1}, \ldots, c_{m}\right) \not 0$ such that $P_{i}\left[\sum_{1}^{m} c_{n} M_{n}\right]=0=P_{i}\left[\sum_{1}^{m} \bar{c}_{n} M_{n}^{*}\right]$. Therefore one $P_{i} M_{n}$ is a linear combination of the rest, say

$$
P_{i} M_{k}=\sum_{n \neq k} a_{n} P_{i} M_{n} \text { and } P_{i} M_{n}^{*}=\sum_{n \neq k} \bar{a}_{n} P_{i} M_{n}^{*} .
$$

Hence

$$
\begin{aligned}
P_{i}\left[\sum_{1}^{m} M_{n} X M_{n}\right] & =\sum_{n=1}^{m} P_{i} M_{n} X M_{n}=\sum_{n \neq k} P_{i} M_{n} X M_{n}+P_{i} M_{k} X M_{k} \\
& =\sum_{n \neq k} P_{i} M_{n} X M_{n}+\sum_{n \neq k} a_{n} P_{i} M_{n} X M_{n}=\sum_{n \neq k}\left(1+a_{n}\right) P_{i} M_{n} X M_{n},
\end{aligned}
$$

and similarly for $P_{i}\left[\Sigma_{1}^{m} M_{n}^{*} X M_{n}^{*}\right]$.

We stated earlier that we are assuming the induction hypothesis that Theorem 1 (Problem 1, respectively) is true for sequences of normal operators fewer than $m$. 
Therefore

$$
\begin{aligned}
\left\|P_{i}\left[\sum_{1}^{m} M_{n} X M_{n}\right] P_{j}\right\|_{2} & =\left\|\sum_{n \neq k}\left(1+a_{n}\right) M_{n}\left(P_{i} X P_{j} h\right) M_{n}\right\|_{2} \\
& =\left\|\sum_{n \neq k}\left(1+\bar{a}_{n}\right) M_{n}^{*}\left(P_{i} X P_{j}\right) M_{n}^{*}\right\|_{2}=\left\|P_{i}\left[\sum_{1}^{m} M_{n}^{*} X M_{n}^{*}\right] P_{j}\right\|_{2}
\end{aligned}
$$

since both sums are the case of a sequence of at most $m-1$ normal operators, so the induction hypothesis applies.

Now the only thing left to consider is the case $i=j=\infty$. Use the operator

$$
P_{\infty}\left[\sum_{1}^{m} M_{n} X M_{n}\right] P_{\infty}=\sum_{1}^{m}\left(P_{\infty} M_{n} P_{\infty}\right)\left(P_{\infty} X P_{\infty}\right)\left(P_{\infty} M_{n} P_{\infty}\right),
$$

and similarly for $P_{\infty}\left[\sum_{1}^{m} M_{n}^{*} X M_{n}^{*}\right] P_{\infty}$. In other words this is precisely the kind of operator of Theorem 1 (Problem 1) and Lemma 4 but with all the operators viewed as acting on $L^{2}\left(E_{\infty}\right)$ (since $P_{\infty} Y P_{\infty} \in L\left(L^{2}\left(E_{\infty}\right)\right)$ ).

By virtue of the essential claim mentioned earlier in this proof, there is no measurable set $E \subset E_{\infty}$ with $\mu E>0$ on which $\sum_{1}^{m} c_{n} \phi_{n}(x)=0$ for some fixed $\left(c_{1}, \ldots, c_{m}\right) \neq 0$. Since $i=j=\infty$ is the only case of Theorem 1 not yet proven, we therefore have reduced to the case of Lemma 8. Q.E.D.

Lemma 9. Theorem 1 (Problem 1) is equivalent to the case $H=L^{2}[0,1]$, and for each $n, M_{n}=M_{\phi_{n}}$ where $\phi_{n} \in L^{\infty}[0,1]$ with Lebesgue measure as the underlying measure.

Proof. By Lemmas 7 and $8, H=L^{2}(\mu), \phi_{n} \in L^{2}(\mu)$ where $(X, \mu)$ is a regular borel probability measure on a compact Hausdorff space, and for all $\left(c_{1}, \ldots, c_{m}\right) \neq 0$, $\mu\left\{x \in X: \sum_{1}^{m} c_{n} \phi_{n}(x)=0\right\}=0$.

In a regular borel probability measure space, on a compact Hausdorff space it is well known that the only atoms are points. We claim that $(X, \mu)$ contain no atoms. Suppose $\left\{x_{0}\right\}$ is an atom. Therefore $\mu\left\{x_{0}\right\}>0$. Therefore if $\phi_{1}\left(x_{0}\right) \neq 0$, then $\left(\phi_{2}\left(x_{0}\right) / \phi_{1}\left(x_{0}\right)\right) \phi_{1}\left(x_{0}\right)-\phi_{2}\left(x_{0}\right)=0 ;$ if $\phi_{2}\left(x_{0}\right) \neq 0$, then $\phi_{1}\left(x_{0}\right)-$ $\left(\phi_{1}\left(x_{0}\right) / \phi_{2}\left(x_{0}\right)\right) \phi_{2}\left(x_{0}\right)=0$; and if $\phi_{1}\left(x_{0}\right)=\phi_{2}\left(x_{0}\right)=0$, then $\phi_{1}\left(x_{0}\right)+\phi_{2}\left(x_{0}\right)=0$. In any of these cases, the atom is a set of positive measure on which $\left\langle\phi_{n}\right\rangle_{1}^{m}$ are linearly dependent. This contradicts the condition of Lemma 8.

By the well-known Carathéodory measure isomorphism theorem, $(X, \mu)$ is measure-theoretically equivalent to Lebesgue measure on $[0,1]$. Therefore we can substitute $([0,1], m)$ for $(X, \mu)$ in the conditions of Lemma 7 .

Proposition 10. If $\left\langle P_{k}\right\rangle_{1}^{\infty}$ is a sequence of projection operators such that $P_{k} \rightarrow I$ in the strong operator topology, and $Y \in L(H)$, then $Y \in C_{2}$ if and only if for some $M<\infty,\left\|P_{k} Y P_{k}\right\|_{2} \leqslant M$. In case $Y \in C_{2}$, we have $\|Y\|_{2}=\lim _{k \rightarrow \infty}\left\|P_{k} Y P_{k}\right\|_{2}$. 
Proof. This is a standard fact. For a short proof, let $\left\langle e_{n}\right\rangle_{1}^{\infty}$ be any basis of $H$. Then for all $N$,

$$
\sum_{1 \leqslant i, j \leqslant N}\left|\left(Y e_{i}, e_{j}\right)\right|^{2}=\lim _{k \rightarrow \infty} \sum_{1 \leqslant i, j \leqslant N}\left|\left(P_{k} Y P_{k} e_{i}, e_{j}\right)\right|^{2} \leqslant M .
$$

$N$ is arbitrary, hence $\|Y\|_{2} \leqslant M$. The rest is straightforward. Q.E.D.

LEMma 11. Theorem 1 (Problem 1) is equivalent to Lemma 9 where, in addition, we assume each $\phi_{n}(x)$ is continuous.

Proof. Using Lusin's Theorem repeatedly on the $\phi_{n}$ 's, we can obtain a closed set $F_{k} \subset[0,1]$ on which all the $\phi_{n}$ 's are continuous and $m\left([0,1] \backslash F_{k}\right)<1 / k$. Hence if we set $P_{k}=M \chi_{E_{k}}$, then each $P_{k}$ commutes with each $M_{\phi_{n}}$ and $P_{k} \rightarrow I$ in the strong operator topology.

Now extend each $\phi_{n}$ on $F_{k}$ continuously to all of $[0,1]$, and call this extension $\Psi_{n}$. Then $P_{k} M_{\phi_{n}}=P_{k} M_{\Psi_{n}}$. Therefore

$$
P_{k}\left[\sum_{1}^{m} M_{n} X M_{n}\right] P_{k}=\sum_{1}^{m} M_{\Psi_{n}}\left(P_{k} X P_{k}\right) M_{\Psi_{n}}
$$

and

$$
P_{k}\left[\sum_{1}^{m} M_{n}^{*} X M_{n}^{*}\right] P_{k}=\sum_{1}^{m} M_{\Psi_{n}}^{*}\left(P_{k} X P_{k}\right) M_{\Psi_{n}}^{*} .
$$

The right-hand sides of the last two equations satisfy the additional hypothesis of Lemma 11. Hence assuming Theorem 1 (Problem 1) true in this case implies that the left-hand sides of those equations have equal Hilbert-Schmidt norms. Then applying Proposition 10 we obtain Theorem 1 for the $M_{\phi_{n}}$ 's. Q.E.D.

Summary. Theorem 1 (Problem 1) is equivalent to the case $H=L^{2}[0,1], M_{n}=N_{n}$ $=M_{\phi_{n}}$ where $\phi_{n} \in L^{\infty}[0,1]$ for each $n$, with the property that

$$
m\left\{x \in[0,1]: \sum_{1}^{m} c_{n} \phi_{n}(x)=0\right\}=0
$$

for every $m$-tuple $\left(c_{1}, \ldots, c_{m}\right) \neq 0$.

Definition. Set $O=\left\{(x, y) \in(0,1) \times(0,1): \sum_{1}^{m} \phi_{n}(x) \phi_{n}(y) \neq 0\right\}$. The set $O$ is open in $[0,1] \times[0,1]$ by the continuity of the $\phi_{n}$ 's.

Proposition 12. $m \times m[[0,1] \times[0,1] \backslash O]=0$.

Proof. Suppose not. Then by Tonelli's Theorem there exists a cross-section $E$ of positive measure. That is, there exists $y \in[0,1]$ such that $\sum_{1}^{m} \phi_{n}(x) \phi_{n}\left(y_{0}\right)=0$ on $E$. This contradicts the condition in the summary on the $\phi_{n}$ 's.

LEMMA 13. Let $\left\langle\phi_{n}(x)\right\rangle_{1}^{m},\left\langle\Psi_{n}(x)\right\rangle_{1}^{m}$ be sequences in $L^{\infty}(0,1)$. Let $H=L^{2}(0,1)$. Define for each $X \in L(H)$, the operator $\Delta(X)=\Sigma_{1}^{m} M_{\phi_{n}} X M_{\Psi_{n}}$ which maps $L(H) \rightarrow$ $L(H)$. If $X$ is a Hilbert-Schmidt operator, and so it has a kernel representation $F(x, y)$ where $(X f)(x)=\int F(x, y) f(y) d y$, then $\Delta(X)$ is a Hilbert-Schmidt operator with the kernel function $\left[\Sigma_{1}^{m} \Phi_{n}(x) \Psi_{n}(y)\right] F(x, y)$. 
Proof. Simply compute $\Delta(X)$ using the kernel representation for $X$ to obtain the kernel function for $\Delta(X)$. The case $X=f \otimes g, F(x, y)=f(x) \overline{g(y)}$ is easiest to check. The Schmidt representation $X=\Sigma \lambda_{n} f_{n} \otimes g_{n}$ completes the proof [2].

Lemma 14 (The Key Lemma). Suppose $\sum_{1}^{m} M_{\phi_{n}} X M_{\phi_{n}} \in C_{2}$ where $H=L^{2}(0,1)$, $\left\langle\phi_{n}(x)\right\rangle_{1}^{m} \subset L^{\infty}(0,1)$ and $X \in L(H)$. Also suppose for every $\left(c_{1}, \ldots, c_{m}\right) \not 0$, $m\left\{x: \sum_{1}^{m} c_{n} \phi_{n}(x)=0\right\}=0$. Then for every $\left(x_{0}, y_{0}\right) \in O$, there exist arbitrarily small open intervals $I, J$ where $x_{0} \in I, y_{0} \in J$ such that $M_{x_{I}} X M_{x_{J}} \in C_{2}$.

Proof. Let $\left(x_{0}, y_{0}\right) \in O$. Set $\Sigma_{1}^{m} \phi_{n}\left(x_{0}\right) \phi_{n}\left(y_{0}\right)=a \neq 0$. Then

$$
\sum_{1}^{m} \phi_{n}(x) \phi_{n}(y)=a\left[1-\frac{\sum_{1}^{m} \phi_{n}(x) \phi_{n}(y)-a}{a}\right] .
$$

By the continuity of $\sum_{1}^{m} \phi_{n}(x) \phi_{n}(y)$ and the fact that $a \neq 0$, there exist arbitrarily small open intervals $I, J$ with $x_{0} \in I$ and $y_{0} \in J$ such that for every $(x, y) \in I \times J$,

$$
\left|\frac{\sum_{1}^{m} \phi_{n}(x) \phi_{n}(y)-a}{a}\right| \leqslant \varepsilon,
$$

$\varepsilon$ to be determined later, but for now at least $\varepsilon<1$. We shall prove that for $\varepsilon$ sufficiently small, the operator $M_{\chi_{I}} X M_{\chi_{J}} \in C_{2}$.

Define $\delta: L(H) \rightarrow L(H)$ by

$$
\delta(X)=\frac{1}{a}\left[\sum_{1}^{m} M_{\phi_{n}} X M_{\phi_{n}}-a X\right] .
$$

A direct computation shows that if $\Delta(X)=\Sigma_{1}^{m} M_{\phi_{n}} X M_{\phi_{n}}$ then

$$
\Delta=a(I-\delta)
$$

where $\Delta, \delta, I$ (the identity) are viewed as maps on $L(H) \rightarrow L(H)$.

It is clear from its definition that $\delta: C_{2} \rightarrow C_{2}$. We further claim that the map $\gamma$ : $X \rightarrow \delta\left(M_{\chi_{1}} X M_{\chi_{J}}\right)$ is a contraction on $C_{2} \rightarrow C_{2}$ of norm bounded by $\varepsilon$. To see this, if $X \in C_{2}$ let $F(x, y)$ denote the kernel function that reproduces $X$. By Lemma 13, the kernel function for $\gamma(X)$ is

$$
G(x, y)=\frac{1}{a}\left(\sum_{1}^{m} \phi_{n}(x) \phi_{n}(y)-a\right) F(x, y) \chi_{I}(x) \chi_{J}(y) .
$$

But then

$$
\left\|\delta\left(M_{\chi_{I}} X M_{\chi_{J}}\right)\right\|_{2}^{2}=\iint|G|^{2} \leqslant \varepsilon^{2} \iint|F|^{2}=\varepsilon^{2}\|X\|_{2}^{2}
$$

because $\left|a^{-1}\left(\Sigma_{1}^{m} \phi_{n}(x) \phi_{n}(y)-a\right)\right| \leqslant \varepsilon<1$ on $I \times J$. Therefore $\gamma$ is a contraction on $C_{2}$ of norm less than or equal to $\varepsilon$. We have

$$
M_{\chi_{I}} \Delta(X) M_{\chi_{J}}=\Delta\left(M_{\chi_{I}} X M_{\chi_{J}}\right)=a(I-\delta)\left(M_{\chi_{I}} X M_{\chi_{J}}\right) \text {. }
$$

The hypothesis of Lemma 11 is that $\Delta(X) \in C_{2}$. Hence $(I-\delta)\left(M_{\chi_{I}} X M_{\chi_{J}}\right) \in C_{2}$. Set $\eta=\left\|(I-\delta)\left(M_{\chi_{1}} X M_{\chi_{J}}\right)\right\|_{2}$. We also have

$$
\gamma^{n}(I-\delta)\left(M_{\chi_{I}} X M_{\chi_{J}}\right)=\delta^{n}(I-\delta)\left(M_{\chi_{I}} X M_{\chi_{J}}\right)
$$


since $M_{\chi}, M_{\chi}$ are projection operators and commute with $\delta$. Therefore

$$
\left\|\delta^{n}(I-\delta)\left(M_{\chi_{1}} X M_{\chi_{J}}\right)\right\|_{2} \leqslant \varepsilon^{n} \eta
$$

If $k>n$ then

$$
\begin{aligned}
\left(I-\delta^{k}\right)\left(M_{\chi_{l}} X M_{\chi_{J}}\right) & -\left(I-\delta^{n}\right)\left(M_{\chi_{I}} X M_{\chi_{J}}\right) \\
= & \left(\delta^{n}-\delta^{k}\right)\left(M_{\chi_{l}} X M_{\chi_{J}}\right)=\left(\sum_{n}^{k-1} \delta^{i}\right)(1-\delta)\left(M_{\chi_{I}} X M_{\chi_{J}}\right) .
\end{aligned}
$$

This operator has Hilbert-Schmidt norm bounded above by

$$
\left(\sum_{n}^{k-1} \varepsilon^{i}\right) \eta<\varepsilon^{n}(1-\varepsilon)^{-1} \eta \rightarrow 0 \quad \text { as } n \rightarrow \infty
$$

Also

$$
\left(I-\delta^{n}\right)\left(M_{\chi_{I}} X M_{\chi_{J}}\right)=\left(\sum_{0}^{n-1} \delta^{\eta}\right)(I-\delta)\left(M_{\chi_{I}} X M_{\chi_{J}}\right) \in C_{2}
$$

(here we let $\delta^{0}$ denote the identity map on $\left.L(H)\right)$. Hence the sequence of operators $\left(I-\delta^{\eta}\right)\left(M_{\chi_{1}} X M_{\chi_{J}}\right)$ form a Cauchy sequence in $C_{2}$. Therefore $\left(I-\delta^{\eta}\right)\left(M_{\chi_{1}} X M_{\chi_{J}}\right)$ $\rightarrow K$ in the $C_{2}$ norm and $K \in C_{2}$.

Set $Y=M_{\chi_{1}} X M_{\chi_{J}}-K$. Then $Y-\delta^{\eta}\left(M_{\chi_{1}} X M_{\chi_{J}}\right) \rightarrow 0$ in $C_{2}$. That is, $Y=$ $\lim _{\eta \rightarrow \infty} \delta^{\eta}\left(M_{\chi_{I}} X M_{\chi_{J}}\right)$, the limit taken in the uniform operator topology. Then

$$
\gamma(Y)=\gamma \lim _{\eta \rightarrow \infty} \delta^{\eta}\left(M_{\chi_{I}} X M_{\chi_{J}}\right)=\lim _{\eta \rightarrow \infty} \gamma \delta^{\eta}\left(M_{\chi_{I}} X M_{\chi_{J}}\right)=\lim _{\eta \rightarrow \infty} \delta^{\eta+1}\left(M_{\chi_{I}} X M_{\chi_{J}}\right) \text {. }
$$

Therefore $Y=\gamma(Y)$ and so $Y$ is a fixed point of $\gamma$.

It suffices to prove $Y=\gamma(Y)$ implies $Y=0$. Then we would have $M_{\chi_{I}} X M_{\chi_{J}}=K$ $\in C_{2}$. This is the part of the proof where we need to choose $\varepsilon$ sufficiently small in order to force $\gamma$ to fail to possess a nonzero fixed point.

Notice that if $R$ is a rank one operator then $M_{\chi_{l}} R M_{\chi_{J}}$ is also rank 1 and by the definition of $\delta, \gamma(R)=\delta\left(M_{\chi_{l}} R M_{\chi_{J}}\right)$ has rank at most $m+1$. Hence $\gamma^{n}(R)$ has rank at most $(m+1)^{n}$.

Notice that if $A$ is a rank $k$ operator then $\|A\| \leqslant \sqrt{k}\|A\|_{2}$. This follows easily from Hölder's inequality applied to the diagonal sequences of $|A|$, which can be nonzero in at most $k$ entries. Recall again that if $A \in L\left(L^{2}(0,1)\right)$ and $f, g \in L^{2}(0,1)$, then $(A f, g)=\operatorname{trace} A(f \otimes g)$ when $f \otimes g$ is the rank one operator $h \rightarrow(h, g) f$. Also if $\phi, \Psi \in L^{\infty}(0,1)$ then

$$
\begin{aligned}
M_{\phi}(f \otimes g) M_{\Psi}(h) & =\phi[(f \otimes g)(\Psi h)]=(\Psi h, g) \phi f \\
& =(h, \bar{\Psi} g) \phi f=(\phi f \otimes \bar{\Psi} g)(h),
\end{aligned}
$$

for every $h \in L^{2}(0,1)$. In other words $M_{\phi}(f \otimes g) M_{\phi}=\phi f \otimes \bar{\Psi} g$.

Finally recall that if $R$ is a trace class operator and $A \in L(H)$ then, as is well known, $\operatorname{Tr} A R=\operatorname{Tr} R A$. Letting $A=M_{n}, R=X M_{n}(f \otimes g)$, we obtain

$$
\operatorname{tr} M_{n} X M_{n}(f \otimes g)=\operatorname{tr} X M_{n}(f \otimes g) M_{n} .
$$


From this it follows that $\operatorname{tr} \delta(A)(f \otimes g)=\operatorname{tr} A \delta(f \otimes g)$. Indeed $\operatorname{tr} \delta^{n}(A)(f \otimes g)=$ $\operatorname{tr} A \delta^{n}(f \otimes g)$.

Putting all this together, we have that if $f, g \in L^{2}(0,1)$ and $\gamma(Y)=Y$, then $Y=\gamma^{n}(Y)$. Hence

$$
\begin{aligned}
(Y f, g) & =\left(\gamma^{n}(Y) f, g\right)=\left(\delta^{n}\left(M_{\chi_{I}} Y M_{\chi_{J}}\right) f, g\right)=\operatorname{tr} \delta^{n}\left(M_{\chi_{I}} Y M_{\chi_{J}}\right)(f \otimes g) \\
& =\operatorname{tr}\left[M_{\chi_{I}} Y M_{\chi_{J}}\right] \delta^{n}(f \otimes g)=\operatorname{tr}\left[Y M_{\chi_{J}} \delta^{n}(f \otimes g) M_{\chi_{I}}\right] .
\end{aligned}
$$

Hence

$$
\begin{aligned}
|(Y f, g)| & =\left|\operatorname{tr}\left[Y M_{\chi_{J}} \delta^{\eta}(f \otimes g) M_{\chi_{I}}\right]\right|=\left|\operatorname{tr}\left[Y \delta^{n}\left(M_{\chi_{J}}(f \otimes g) M_{\chi_{I}}\right)\right]\right| \\
& \leqslant\|Y\|_{L(H)}\left\|\delta^{\eta}\left(M_{\chi_{J}}(f \otimes g) M_{\chi_{I}}\right)\right\|_{1} \\
& \leqslant\|Y\|_{L(H)} \cdot \sqrt{(m+1)^{n}}\left\|\delta^{\eta}\left(M_{\chi_{J}}(f \otimes g) M_{\chi_{I}}\right)\right\|_{2} .
\end{aligned}
$$

A straightforward verification shows that the $L^{2}$-kernel function for the operator $f \otimes g$ is $f(x) \overline{g(y)}$. Indeed

$$
((f \otimes g)(h), k)=\iint h(y) \overline{g(y)} f(x) \overline{k(x)} .
$$

Therefore the kernel function for $\delta^{n}\left(M_{\chi_{J}}(f \otimes g) M_{\chi_{I}}\right)=\delta^{n}\left(\chi_{J} f \otimes \chi_{I} g\right)$, by Lemma 13 , is

$$
H(x, y)=\left(\frac{1}{a}\left[\sum_{1}^{m} \phi_{n}(x) \phi_{n}(y)-a\right]\right)^{n} \chi_{J}(x) f(x) \chi_{I}(y) \overline{g(y)} .
$$

But $\left|a^{-1}\left[\sum_{1}^{m} \phi_{n}(x) \phi_{n}(y)-a\right]\right| \leqslant \varepsilon$ on $I \times J$. Therefore

$$
|H(x, y)| \leqslant \varepsilon^{\eta}|f(x)||g(y)|
$$

and so

$$
\left\|\delta^{\eta}\left(M_{\chi_{J}}(f \otimes g) M_{\chi_{I}}\right)\right\|_{2}=\left[\iint|H(x, y)|^{2}\right]^{1 / 2} \leqslant \varepsilon^{\eta}\|f\|\|g\| .
$$

Hence

$$
\begin{aligned}
|(Y f, g)| & \leqslant\|Y\|_{L(H)} \sqrt{(m+1)^{n}}\left\|\delta^{\eta}\left(M_{\chi_{J}}(f \otimes g) M_{\chi_{I}}\right)\right\|_{2} \\
& \leqslant\|Y\|_{L(H)} \sqrt{(m+1)^{n}} \cdot \varepsilon^{\eta}\|f\|\|g\| .
\end{aligned}
$$

Now choose $\varepsilon=1 / 2 \sqrt{m+1}$. Then $|(Y f, g)| \leqslant\|y\|_{L(H)} 2^{-n}\|f\|\|g\|$. Since $n$ is arbitrary, we have that $(Y f, g)=0$. Since $f, g \in L^{2}(0,1)$ were arbitrary, $Y=0$ and hence $M_{\chi_{I}} X M_{\chi_{J}}=K \in C_{2}$. Q.E.D.

LEMMA 15. There exist disjoint open squares $I_{n} \times J_{n}$ in $(0,1) \times(0,1)$ such that $m \times m\left(\cup I_{n} \times J_{n}\right)=1$ and $M_{\chi_{I_{n}}} X M_{\chi_{J_{n}}} \in C_{2}$ for each $n$.

Proof. By Lemma 14, for each $\left(x_{0}, y_{0}\right) \in O$, there exist arbitrarily small open squares $I \times J \subset O$ centered at $\left(x_{0}, y_{0}\right)$ such that $M_{\chi_{I}} X M_{\chi_{J}} \in C_{2}$. The collection of all such open squares must therefore form a Vitali covering of $O$, which has finite Lebesgue measure in the plane. 
By the Vitali covering lemma, taking $\varepsilon=\frac{1}{2}$, there exists a finite disjoint collection of these open squares $\left\{I_{k} \times J_{k}\right\}_{k=1}^{n_{1}}$ such that $m \times m\left(O \backslash \cup \overline{I_{k} \times J_{k}}\right)<\frac{1}{2}$. Again by

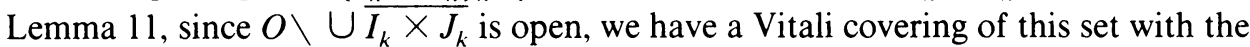
same kind of open squares having the same properties as before. Choosing $\varepsilon=\frac{1}{4}$ and applying the Vitali covering lemma again, we obtain a finite disjoint collection of open squares $\left\{I_{k} \times J_{k}\right\}_{n_{1}+1}^{n_{2}}$ such that $m \times m\left(O \backslash \cup_{1}^{n_{2}} I_{k} \times J_{k}\right)<\frac{1}{4}$ and $M \chi_{I_{n}} X M \chi_{J_{n}}$ $\in C_{2}$, and so on for $\varepsilon=\frac{1}{8}, \frac{1}{16}, \ldots$ This produces our sequences of open squares $I_{n} \times J_{n} \subset O$ such that $m \times m\left(O \backslash \cup I_{n} \times J_{n}\right)=0$ and $M \chi_{I_{n}} X M \chi_{J_{n}} \in C_{2}$ for all $n$. Q.E.D.

REMARKS. Another proof of this lemma exists without using the Vitali covering lemma. Use a grid argument to obtain a closed set in $O$ with planar measure approximately 1 . Then use compactness to obtain a finite cover consisting of the desired open squares. Then consider all the overlappings and extract the interiors of the components. Subtract off their closures from $O$ and you obtain another open set. Do this all again, and again. This, in the limit, exhausts all of $O$ (not merely a subset of $O$ of planar measure 1).

Lemma 16. Define $\Delta(X)=\sum_{1}^{m} M_{\phi_{n}} X M_{\phi_{n}}$ and $\Delta^{*}(X)=\sum_{1}^{m} M_{\phi_{n}}^{*} X M_{\phi_{n}}^{*}$, where $\left\langle\phi_{n}\right\rangle_{1}^{m}$ $\subset L^{\infty}(0,1)$ and $X \in L\left(L^{2}(0,1)\right)$. Suppose $\left\langle I_{k} \times J_{k}\right\rangle$ is a sequence of disjoint open squares for which

(i) $m \times m\left(\cup I_{k} \times J_{k}\right)=1$ and

(ii) $M \chi_{I_{k}} X M \chi_{J_{k}} \in C_{2}$ for every $k$.

Let $P_{k}=M \chi_{I_{k}}$ and $Q_{k}=M \chi_{J_{k}}$. If $\Delta(X) \in C_{2}$, then

(a) $\Delta(X)=\sum P_{k} \Delta(X) Q_{k}$, where this sum converges in $C_{2}$.

(b) $\|\Delta(X)\|_{2}^{2}=\Sigma_{k}\left\|P_{k} \Delta(X) Q_{k}\right\|_{2}^{2}$. (More generally if $K \in C_{2}$ then $\|K\|_{2}^{2}=$ $\Sigma_{k}\left\|P_{k} K Q_{k}\right\|_{2}^{2}$.)

(c) $\left\|P_{k} \Delta(X) Q_{k}\right\|_{2}=\left\|P_{k} \Delta^{*}(X) Q_{k}\right\|_{2}$.

(d) $\Sigma_{k}\left\|P_{k} \Delta^{*}(X) Q_{k}\right\|_{2}^{2}<\infty$.

Proof. To prove part (a) let $F(x, y) \in L^{2}((0,1) \times(0,1))$ denote the kernel function for $\Delta(X)$. Then

$$
\begin{aligned}
\left\|\Delta(X)-\sum_{1}^{N} P_{k} \Delta(X) Q_{k}\right\|_{2}^{2} & =\iint\left|\left(1-\sum_{1}^{N} \chi_{I_{n}}(x) \chi_{J_{n}}(y)\right) F(x, y)\right|^{2} \\
& \rightarrow 0 \text { as } N \rightarrow \infty
\end{aligned}
$$

by the Lebesgue dominated convergence theorem. Indeed $m \times m\left(\biguplus I_{n} \times J_{n}\right)=1$ implies $\sum_{1}^{N} \chi_{I_{n}}(x) \chi_{J_{n}}(y) \rightarrow 1$ a.e. in $[0,1] \times[0,1]$ and the integral is dominated by $|F(x, y)|^{2}$.

To prove (b), notice that

$$
\begin{aligned}
\sum_{k}\left\|P_{k} \Delta(X) Q_{k}\right\|_{2}^{2} & =\sum_{k} \iint\left|\chi_{I_{n}}(x) \chi_{J_{n}}(y) F(x, y)\right|^{2} \\
& =\iint_{\cup\left(I_{n} \times J_{n}\right)}|F|^{2}=\iint|F|^{2}=\|\Delta(X)\|_{2}^{2} .
\end{aligned}
$$


To prove (c) notice that $P_{k}, Q_{k}$ commutes with $\Delta$ and $\Delta^{*}$. By hypothesis (ii) $P_{k} X Q_{k} \in C_{2}$ for all $k$. By Corollary 2

$$
\left\|P_{k} \Delta(X) Q_{k}\right\|_{2}=\left\|\Delta\left(P_{k} X Q_{k}\right)\right\|_{2}=\left\|\Delta^{*}\left(P_{k} X Q_{k}\right)\right\|_{2}=\left\|P_{k} \Delta^{*}(X) Q_{k}\right\|_{2} .
$$

To prove (d) observe that by (b) and (c),

$$
\sum\left\|P_{k} \Delta^{*}(X) Q_{k}\right\|_{2}^{2}=\sum\left\|P_{k} \Delta(X) Q_{k}\right\|^{2}=\|\Delta(X)\|_{2}^{2}<\infty \text {. Q.E.D. }
$$

Proof of Theorem 1. If $\Delta(X), \Delta^{*}(X) \in C_{2}$ then Lemma 16(b), (c) apply to give

$$
\|\Delta(X)\|_{2}^{2}=\sum\left\|P_{k} \Delta(X) Q_{k}\right\|_{2}^{2}=\sum\left\|P_{k} \Delta^{*}(X) Q_{k}\right\|_{2}^{2}=\left\|\Delta^{*}(X)\right\|_{2}^{2} \text {. Q.E.D. }
$$

The last theorem of this section connects (a) and (b) in Problem 1. We find it striking and a bit curious.

Theorem 17. If $\Delta, \Delta^{*}$ satisfy the property in Problem 1(a), that is, that $\Delta(X)=0$ imply $\Delta^{*}(X)=0$, then $\Delta(X) \in C_{2}$ implies $\Delta^{* 2}(X) \in C_{2}$ with $\left\|\Delta^{2}(X)\right\|_{2}=$ $\left\|\Delta^{* 2}(X)\right\|_{2}$.

Proof. Let $T=\Delta^{*}(X)-\Sigma_{k} P_{k} \Delta^{*}(X) Q_{k}$. Then

$$
\begin{aligned}
\Delta(T) & =\Delta \Delta^{*}(X)-\Delta\left(\sum_{k} P_{k} \Delta^{*}(X) Q_{k}\right)=\Delta^{*} \Delta(X)-\sum_{k} P_{k} \Delta^{*}(\Delta(X)) Q_{k} \\
& =\Delta^{*}\left[\Delta(X)-\sum_{k} P_{k} \Delta(X) Q_{k}\right]=0 .
\end{aligned}
$$

By Lemma 16(a), since $\Delta T=0$, we have $0=\Delta^{*} T=\Delta^{* 2}(X)-\sum P_{k} \Delta^{* 2}(X) Q_{k}$. But $\sum P_{k} \Delta^{*}(X) Q_{k} \in C_{2}$. Hence

$$
\Delta^{*}\left(\sum_{k} P_{k} \Delta^{*}(X) Q_{k}\right)=\sum P_{k} \Delta^{* 2}(X) Q_{k} \Delta^{* 2}(X) \in C_{2} .
$$

This almost completes the proof. What is incomplete is that previously we used the induction hypotheses relating $\Delta$ and $\Delta^{*}$ to make reductions to the present case for Theorem 1 . The reader must consider the previous reductions using the different hypothesis that Theorem 17 relating $\Delta$ and $\Delta^{* 2}$ holds true for $\Delta$ sums of size less than $m$. That $\left\|\Delta^{2}(X)\right\|_{2}=\left\|\Delta^{* 2}(X)\right\|_{2}$ follows from Theorem 1 and the first part of this theorem. Q.E.D.

REMarK. All the results of this section apply to Problem 1 in exactly the same way as Theorem 1. The reader must substitute the words Problem 1 wherever Theorem 1 appears. In the proofs, the stronger induction hypotheses and the stronger conclusion present no difficulties.

The difficulty in settling Problem 1 in the affirmative lies, for us, in showing that $\Delta^{*}(X)=\Sigma_{1}^{\infty} P_{k} \Delta^{*}(X) Q_{k}$. This remains open.

There is an alternate proof of Theorem 1 which follows directly from Lemma 8. The disadvantage of it is that it avoids all the constructive aspects of the later proofs, especially the 'local Hilbert-Schmidt' character of $X$. Such local behavior is needed to prove Theorem 17. 
Alternate Proof of Theorem 1. If $\Delta(X), \Delta^{*}(X) \in C_{2}$, letting $G(x, y), H(x, y)$ $\in L^{2}(\mu \times \mu)$ denote their respective reproducing kernels, let $\Delta(x, y)=$ $\sum_{1}^{n} \phi_{n}(x) \phi_{n}(y)$. Since $\Delta$ and $\Delta^{*}$ commute we have that

$$
\frac{\overline{\Delta(x, y)}}{\Delta(x, y)} G(x, y)=\frac{1}{\Delta(x, y)}[\overline{\Delta(x, y)} G]=\frac{1}{\Delta(x, y)}[\Delta(x, y) H]=H(x, y),
$$

where it is elementary to verify that $\overline{\Delta(x, y)} G=\Delta(x, y) H$. Here Lemma 8 is used along with Tonelli's Theorem to insure that $\Delta(x, y) \neq 0 \mu \times \mu$-almost everywhere. Therefore $|G|=|H| \mu \times \mu$-almost everywhere. The rest is clear. Q.E.D.

5. Partial results on the Fuglede Theorem for $\Delta$ modulo the trace class. The following questions appear in [10,9] and remain unsolved.

Question 1. If $N$ is normal and $X \in L(H)$, does $N X-X N \in C_{1}$ imply $N^{*} X-$ $X N^{*} \in C_{1}$ ?

Question 2. If $N$ is normal and $X \in K(H)$, does $N X-X N \in C_{1}$ imply $N^{*} X-$ $X N^{*} \in C_{1}$ ?

Question 3. If $N$ is normal, $X \in K(H)$ does $N X-X N \in C_{1}$ imply $\operatorname{tr}(N X-X N)$ $=0$ ?

In [8, Theorem 8] we proved Question 3 holds true under the additional hypothesis that $X \in C_{2}$. In [10] we proved that if the answer to Question 2 is yes, then it follows that the answer to Question 3 is yes.

Since the Fuglede-Putnam phenomena modulo the Hilbert-Schmidt class holds in a more general setting relating finite sequences of commuting normal operators, and since the Voiculescu phenomena mentioned earlier fail to extend to finite sequences of commuting normal operators, it is natural to ask if [8, Theorem 8] extends to a sum in some way. The theorem states that if $N$ is normal, $X \in C_{2}$ and $N X-X N \in$ $C_{1}$, then $\operatorname{tr}(N X-X N)=0$. For finite sums $\Sigma_{n} N_{n} X-X N_{n}$, the same result holds true for obvious reasons (provided $\left\langle N^{n}\right\rangle$ are commuting normals). In order to find a nontrivial analog to this theorem, the author asked the following unpublished question. If $N_{1}, N_{2}$ are commuting normals and $X_{1}, X_{2} \in C_{2}$ such that $K=N_{1} X_{1}$ $X_{1} N_{1}+N_{2} X_{2}-X_{2} N_{2} \in C_{1}$, must $\operatorname{Tr} K=0$ ? Unfortunately the answer to this question is no. The example is due to recent joint work with Dan Timotyn. We do not include the example in this paper, owing to its length and technical nature.

On an analog to Question 1. It is elementary, though tedious, to produce two $4 \times 4$ matrices, $N$ a diagonal and $X$ nonzero in the $(1,3),(1,4),(2,3),(2,4)$ positions such that $\|N X-X N\|_{1} \neq\left\|N^{*} X-X N^{*}\right\|_{1}$. This suggests that the answer to Question 1 may be no. However, there is an analog to Question 1 that is true.

Consider the $\Delta, \Delta^{*}$ operators on $L(H)$ where, in addition, $N_{n}=M_{n}^{*}$ and $\left\langle M_{n}\right\rangle_{1}^{m}$ are commuting normal operators. That is,

$$
\Delta(X)=\sum_{1}^{m} M_{n} X M_{n}^{*} \quad \text { and } \quad \Delta^{*}(X)=\sum_{1}^{m} M_{n}^{*} X M_{n} .
$$

Clearly $\Delta$ preserves selfadjointness and positivity.

THeorem 18. Let $\Delta$ be as defined above (with $N_{n}=M_{n}^{*}$ ) and $K \in C_{1}$. Then $\operatorname{tr}(\Delta(K))=\operatorname{tr}\left(\Delta^{*}(K)\right)$. In particular, if $K \geqslant 0$ then $\|\Delta(K)\|_{1}=\left\|\Delta^{*}(K)\right\|_{1}$. 
Proof. If $K \geqslant 0$ then $\Delta(K), \Delta^{*}(K) \geqslant 0$ and so $\|\Delta(K)\|_{1}=\operatorname{tr}(\Delta(K))$ and the same for $\Delta^{*}$. Therefore it suffices to prove the first part of the theorem, namely that $\operatorname{tr}(\Delta(K))=\operatorname{tr}\left(\Delta^{*}(K)\right)$ for every $K \in C_{1}$. Clearly then it suffices to prove $\operatorname{tr} M K M^{*}$ $=\operatorname{tr} M^{*} K M$ for every normal operator $M$.

Indeed, more is true. if $A$ commutes with $B$, then $\operatorname{tr} A K B=\operatorname{tr} B K A$, because $\operatorname{tr}$ $A K B=\operatorname{tr} K B A=\operatorname{tr} K A B=\operatorname{tr} B K A$ (using the fact that $\operatorname{tr} T S=\operatorname{tr} S T$ if $S \in L(H)$ and $\left.T \in C_{1}\right)$.

Corollary 19. Let $K \in C_{1}$ and $A_{n}, B_{n} \in L(H)$ such that $A_{n} B_{n}=B_{n} A_{n}$ for $1 \leqslant n \leqslant m$. Then

$$
\operatorname{tr} \sum_{1}^{m} A_{n} K B_{n}=\operatorname{tr} \sum_{1}^{m} B_{n} K A_{n} .
$$

6. The $\Delta$-operator as an infinite sum. In this section we consider the $\Delta$-operator with $m=\infty$. In order to insure convergence of the sum, we shall assume $\sum_{n=1}^{\infty}\left\|M_{n}\right\|\left\|N_{n}\right\|<\infty$. Of course also $\left\langle M_{n}\right\rangle$ and $\left\langle N_{n}\right\rangle$ are still separately commuting sequences of operators. Then

$$
\Delta(X)=\sum_{n=1}^{\infty} M_{n} X N_{n} \text { and } \Delta^{*}(X)=\sum_{n=1}^{\infty} M_{n}^{*} X N_{n}^{*}
$$

are bounded linear operators on $L(H) \rightarrow L(H)$ with $\|\Delta\| \leqslant \sum_{n=1}^{\infty}\left\|M_{n}\right\|\left\|N_{n}\right\|$ and the same for $\Delta^{*}$ (in fact this is true for any $\|\Delta\|_{I}$ of the type where $I$ is a normed ideal for which $\|A K B\|_{I} \leqslant\|A\|\|B\|\|K\|_{I}$ ).

The general question in this section is which of the results of the earlier sections remain true for the infinite sum. The theme is to try to prove Theorem 1 in this setting.

The main difficulty in extending to the infinite case concerns the function $\Delta(x, y)=\sum \phi_{n}(x) \phi_{n}(y)$. First of all the spectral theorem applies to the entire sequence $\left\langle M_{n}\right\rangle_{1}^{\infty}$ and allows us to keep this action on a finite, regular borel, compact, Hausdorff measure space $(X, \mu)$. Clearly the Berberian $2 \times 2$ matrix trick allows us to assume without loss of generality that $N_{n}=M_{n}$ for every $n$, provided Theorem 1 is what we want to prove. Then $\sum\left\|M_{n}\right\|\left\|N_{n}\right\|<\infty$ implies $\sum\left\|\phi_{n}\right\|_{L^{x}}^{2}$ and so the sum $\Delta(x, y)$ converges absolutely a.e. on $X \times X$. If the space $(X, m)$ has atoms then they must be points since $(X, m)$ is a finite regular, borel measure on a compact, Hausdorff space. Let $X_{0}$ be the collection of these atoms. Then $X_{0}$ can be at most countable since $\mu$ is finite. Splitting $X=X_{0} \bullet\left(X-X_{0}\right)$ and $H=L^{2}\left(X_{0}\right) \oplus$ $L^{2}\left(X-X_{0}\right)$, we reduce $M_{\phi_{n}}=D_{n} \oplus M_{\phi_{n}^{\prime}}$ where $D_{n}$ is diagonal with action on $L^{2}\left(X_{0}\right)$ and $M_{\phi_{n}^{\prime}}$ acts on $L^{2}\left(X-X_{0}\right)$, and where $X \backslash X_{0}$ has no atoms. Comparing the $C_{2}$ norms of the 4 operator matrix entries for $\Delta(X)$ and $\Delta^{*}(X)$, we obtain the expressions $\sum D_{n} X D_{n}, \sum M_{\phi_{n}^{\prime}} X D_{n}, \sum D_{n} X M_{\phi_{n}^{\prime}}$ and $\sum M_{\phi_{n}^{\prime}} X M_{\phi_{n}^{\prime}}$, and their * counterparts. A straightforward matrix computation shows Theorem 1 applies to the case $\sum D_{n} X D_{n}$. For the case $\sum D_{n} X M_{\phi_{n}^{\prime}}$, let $P_{i}$ be the rank one orthogonal projection onto the space spanned by $e_{i}$. If $T \in L(H)$ then $\|T\|_{2}^{2}=\sum\left\|P_{i} T\right\|_{2}^{2}$. Therefore to prove Theorem 1 for this case, it suffices to prove it for

$$
P_{i} \sum D_{n} X M_{\phi_{n}^{\prime}}=\sum \lambda_{n} P_{i} X M_{\phi_{n}^{\prime}}=\sum P_{i} X\left(\lambda_{n} M_{\phi_{n}^{\prime}}\right)=P_{i} X\left(\sum \lambda_{n} M_{\phi_{n}^{\prime}}\right)
$$


where $\lambda_{n}=\left(D_{n} e_{i}, e_{i}\right)$. However it is easy to verify $\left\|P_{i} X N\right\|_{2}=\left\|P_{i} X N^{*}\right\|_{2}$ (see $[10$, 11]), hence Theorem 1 holds for $\sum D_{n} X M_{\phi_{n}^{\prime}}$ and similarly for $\sum M_{\phi_{n}^{\prime}} X D_{n}$. The only case left is $\sum M_{\phi_{n}^{\prime}} X M_{\phi_{n}^{\prime}}$ and this is all acting on a finite measure space with no atoms. Therefore involving the Carathéodory measure isomorphism theorem gives us, as before, the reduction to the $L^{2}[0,1]$ case.

LEMma 20. If $\Delta, \Delta^{*}$ are the infinite sums above, then to prove Theorem 1 for $\Delta, \Delta^{*}$ it suffices to assume the action takes place on $L^{2}[0,1]$ and $\phi_{n} \in C[0,1]$ for every $n$.

Proof. After the above remarks we then use Lusin's Theorem on the sequence $\left\langle\phi_{n}\right\rangle$ and mimic the proof of Lemma 11. Q.E.D.

Now if, as before, we set $O=\{(x, y): \Delta(x, y) \neq 0\}$, then $O$ is open by the continuity of each $\phi_{n}$ and the uniform convergence of $\sum \phi_{n}(x) \phi_{n}(y)$. The central problem is that we do not have a reduction like Lemma 8 regarding the zero set for $\Delta$ and the "linear independence" of the infinite sequence $\left\langle M_{\phi_{n}}\right\rangle$ on sets of finite measure in [0,1]. Therefore we do not get $m \times m(O)=1$. This was crucial in order that $\iint|K(x, y)|^{2}=\|K\|_{2}^{2}$ for all $K \in L^{2}([0,1] \times[0,1])$.

Another difficulty is in trying to obtain Lemma 14 (the key lemma) in this setting. It was central to the proof that $\Delta$ applied to a rank one operator yield a rank $m$ operator. In this setting, the infinite summand does not have this property.

Lemma 13 and the earlier results of that section remain true with the identical proofs. At present, for the case of the infinite summand, all we can achieve modulo $C_{2}$ is the following:

Lemma 21. If $X \in C_{2}$ then $\|\Delta(X)\|_{2}=\left\|\Delta^{*}(X)\right\|_{2}$.

Proof. If $F(x, y)$ is the $L^{2}(X \times X, \mu \times \mu)$ kernel function for $X$, then the kernel functions for $\Delta(X)$ and $\Delta^{*}(X)$ are $\Delta(x, y) F(x, y)$ and $\overline{\Delta(x, y)} F(x, y)$, respectively. Q.E.D.

LEMMA 22. Let $\Delta(X)=\sum_{1}^{\infty} M_{\phi_{n}} X M_{\phi_{n}}, \Delta^{*}(X)=\sum_{1}^{\infty} M_{\phi_{n}}^{*} X M_{\phi_{n}}^{*}$ with $\phi_{n} \in C[0,1]$ for every $n$ and $\Sigma_{1}^{\infty}\left\|\phi_{n}\right\|_{L^{x}}^{2}<\infty$. Let $\Delta(x, y)=\sum \phi_{n}(x) \phi_{n}(y)$, and $O=\{(x, y): \Delta(x, y)$ $\neq 0\}$ (hence $O$ is open). Let $\left\{I_{k} \times J_{k}\right\}$ be any collection of rectangles contained in $O$ with disjoint interiors such that $m \times m\left(O \backslash \cup\left(I_{k} \times J_{k}\right)\right)=0$. Let $P_{k}=M_{\chi_{I_{n}}}$ and $Q_{k}=M_{\chi_{J_{k}}}$ If $\Delta(X)$ and $\Delta^{*}(X) \in C_{2}$ then

$$
\left\|\sum_{k} P_{k} \Delta(X) Q_{k}\right\|_{2}^{2}=\sum_{k}\left\|P_{k} \Delta(X) Q_{k}\right\|_{2}^{2}=\sum_{k}\left\|P_{k} \Delta^{*}(X) Q_{k}\right\|_{2}^{2}=\left\|\sum_{k} P_{k} \Delta^{*}(X) Q_{k}\right\|_{2}^{2} \text {. }
$$

Proof. The 1st and 3rd equalities follow as in the proof of Lemma 16(b). Indeed if $F(x, y) \in L^{2}([0,1] \times[0,1])$ is the kernel function for $\Delta(X)$, then $\left(\Sigma_{k} \chi_{I_{k}}(x) \chi_{J_{k}}(y)\right) F(x, y)$ is the kernel function for $\sum P_{k} \Delta(X) Q_{k}$.

The second equality follows from the fact that $\left\|P_{k} \Delta(X) Q_{k}\right\|_{2}=\left\|P_{k} \Delta^{*}(X) Q_{k}\right\|_{2}$ for every $k$. To see this, let $F(x, y), G(x, y)$ denote the kernel functions of $\Delta(X)$ and $\Delta^{*}(X)$, respectively. Since $\Delta, \Delta^{*}$ commute, the kernel functions of $\Delta \Delta^{*}(X)$ and 
$\Delta^{*} \Delta(X)$, which are $\Delta(x, y) G(x, y)$ and $\overline{\Delta(x, y)} F(x, y)$, are equal almost everywhere $[m \times m]$. But then

$$
\begin{aligned}
& \left\|P_{k} \Delta(X) Q_{k}\right\|_{2}^{2}=\iint \chi_{I_{k}}(x) \chi_{J_{k}}(y)|F|^{2}=\iint \chi_{I_{k}}(x) \chi_{J_{k}}(y)\left|\frac{\Delta(x, y)}{\Delta(x, y)}\right|^{2}|F|^{2} \\
& \quad=\iint \chi_{I_{k}}(x) \chi_{J_{k}}(y) \cdot \frac{1}{|\Delta(x, y)|^{2}}|\bar{\Delta} F|^{2}=\iint \chi_{I_{k}}(x) \chi_{J_{k}}(y) \frac{1}{|\Delta(x, y)|^{2}}|\Delta G|^{2} \\
& =\iint \chi_{I_{k}}(x) \chi_{J_{k}}(y)|G|^{2}=\left\|P_{k} \Delta^{*}(X) Q_{k}\right\|_{2}^{2} \text {. Q.E.D. }
\end{aligned}
$$

\section{REFERENCES}

1. B. Fuglede, A commutativity theorem for normal operators, Proc. Nat. Acad. Sci. U.S.A. 36 (1950), $35-40$.

2. I. C. Gohberg and M. G. Krein, Introduction to the theory of linear nonselfadjoint operators, Transl. Math. Monographs, vol. 18, Amer. Math. Soc., Providence, R. I., 1969.

3. R. L. Moore, An asymptotic Fuglede theorem, Proc. Amer. Math. Soc. 50 (1975), 138-142.

4. C. R. Putnam, On normal operators in Hilbert space, Amer. J. Math. 73 (1951), 357-362.

5. D. Rogers, On Fuglede's theorem and operator topologies, Proc. Amer. Math. Soc. 75 (1979), 32-36.

6. M. Rosenblum, On a theorem of Fuglede and Putnam, J. London Math. Soc. 33 (1958), 376-377.

7. D. Voiculescu, Some results on norm ideal perturbations of Hilbert space operators, INCREST preprint No. 34/1978 ibidem II, INCREST preprint No. 39/1978.

8. Gary Weiss, The Fuglede commutativity theorem modulo the Hilbert-Schmidt class and generating functions for matrix operators. I, Trans. Amer. Math. Soc. 246 (1978), 193-209.

9. Fuglede's commutativity theorem modulo the Hilbert-Schmidt class and generating functions for matrix operators. II, J. Operator Theory 5 (1981), 3-16.

10. $113-118$.

11. , Fuglede's commutativity theorem modulo operator ideals, Proc. Amer. Math. Soc. 83 (1981),

II. , Commutators and operator ideals, Dissertation, Univ. of Michigan, Ann Arbor, 1975.

Department of Mathematical Sciences, University of Cincinnati, Cincinnati, Ohio 45221 